\title{
Effects of Cryopreservation on the Cell Viability, Proliferative Capacity and Neuronal Differentiation Potential of Canine Bone Marrow Stromal Cells
}

\author{
Kazuya EDAMURA $^{1) *}$, Rei NAKANO ${ }^{1)}$, Kyohei FUJIMOTO ${ }^{1)}$, Kenji TESHIMA ${ }^{1)}$, Kazushi ASANO ${ }^{1)}$ and \\ Shigeo TANAKA ${ }^{1)}$ \\ ${ }^{1)}$ Laboratory of Veterinary Surgery, Department of Veterinary Medicine, College of Bioresource Sciences, Nihon University, 1866 \\ Kameino, Fujisawa, Kanagawa 252-0880, Japan
}

(Received 8 June 2013/Accepted 29 November 2013/Published online in J-STAGE 13 December 2013)

ABSTRACT. We investigated the cell viability, proliferative capacity and neuronal differentiation potential of canine bone marrow stromal cells (BMSCs) after cryopreservation. BMSCs were cryopreserved using cryoprotectant solutions with 10\% DMSO and $10 \%$ FBS (DF group) or without DMSO and FBS (DF-free group); fresh BMSCs were used as a control. The cell viability and proliferative capacity of BMSCs were similar in the DF-free and control groups, while those in the DF group were lower. In all groups, BMSCs differentiated into neuron-like cells that stained positive against neuron markers, and the mRNA expression levels of neuron markers increased after neuronal induction. In conclusion, cryopreservation with DF-free cryoprotectant solution did not diminish the cell viability, proliferative capacity or neuronal differentiation potential of canine BMSCs.

KEY WORDS: bone marrow stromal cell, canine, cryopreservation, culture, neuron.

doi: 10.1292/jvms.13-0296; J. Vet. Med. Sci. 76(4): 573-577, 2014

Spinal cord regenerative therapy based on transplantation of canine bone marrow stromal cells (BMSCs) has recently been clinically applied for treatment of severe spinal cord injury (SCI) in dogs $[17,18]$. Generally, utilization of autologous BMSCs requires expansion of these cells in culture, which is why at least $1-3$ weeks are necessary between the time of bone marrow collection and transplantation [17]. An effective long-term preservation technique for canine BMSCs that would maintain cell functions and properties is critical for the timely transplantation of an adequate number of autologous canine BMSCs in dogs with SCI.

The most common and widely used method for the longterm preservation of living cells is cryopreservation. However, stem cells, including BMSCs, are sensitive to freezing and can be damaged by the external and/or intracellular ice crystal formation; the survival rate of cryopreserved stem cells after thawing could therefore be significantly decreased $[4,5]$. Currently, there have been only a few basic researches on cryopreservation of canine BMSCs. In the present study, we investigated the cell viability and proliferative capacity of cryopreserved canine BMSCs after thawing.

Cryoprotectant solutions containing dimethyl sulfoxide (DMSO) are frequently used for cryopreservation of stem cells $[5,12,16]$. However, DMSO can be also cytotoxic and may affect the stem cell capacity for proliferation and differ-

*Correspondence to: Edamura, K., Laboratory of Veterinary Surgery, Department of Veterinary Medicine, College of Bioresource Sciences, Nihon University, 1866 Kameino, Fujisawa, Kanagawa 252-0880, Japan. e-mail: edamura.kazuya@nihon-u.ac.jp

(C)2014 The Japanese Society of Veterinary Science

This is an open-access article distributed under the terms of the Creative Commons Attribution Non-Commercial No Derivatives (by-nc-nd) License $<$ http://creativecommons.org/licenses/by-nc-nd/3.0/>. entiation after thawing $[3,5,13,20]$. In the present study, we therefore compared the effects of cryoprotectant solutions with or without DMSO on the proliferation and neuronal differentiation of canine BMSCs.

The present study was conducted in accordance with the Guidelines for the Care and Use of Laboratory Animals of the College of Bioresource Sciences of Nihon University. Three healthy adult beagles were used for BMSC isolation performed as previously described [2]. Briefly, bone marrow was collected from the humerus under general anesthesia, and mononuclear cells were obtained after separation by density gradient centrifugation using a Histopaque-1077 (Sigma-Aldrich, St. Louis, MO, U.S.A.). The mononuclear cells were transferred to two $25-\mathrm{cm}^{2}$ culture flasks and statically cultured in $\alpha$-modified Eagle's minimum essential medium ( $\alpha$-MEM; Invitrogen, Carlsbad, CA, U.S.A.) supplemented with $10 \%$ fetal bovine serum (FBS; Invitrogen) at $5 \% \mathrm{CO}_{2}$ and $37^{\circ} \mathrm{C}$. Non-adherent cells were removed by aspiration, and the attached canine BMSCs were cultured until almost reached confluency and collected using $0.25 \%$ trypsin-EDTA (Invitrogen).

Canine BMSCs were cryopreserved using cryoprotectant solutions with 10\% DMSO and 10\% FBS (DF group: $n=6$ ) or without DMSO and FBS (Cellvation ${ }^{\circledR}$; Celox Laboratories, St. Paul, MN, U.S.A.) (DF-free group: $n=6)$. Cells (1.0 $\times 10^{6}$ ) were resuspended in $1 \mathrm{~m} l$ of cryoprotectant solution within a cryogenic vial (Sumitomo Bakelite, Tokyo, Japan) and placed in a biofreezing vessel (BICELL ${ }^{\circledR}$; Nihon Freezer, Tokyo, Japan) for cryopreservation at $-80^{\circ} \mathrm{C}$ in a freezer without programmed decrease in temperature. After 7 days of cryopreservation, vials were removed from the biofreezing vessel and immersed in a water bath at $37^{\circ} \mathrm{C}$ for $1 \mathrm{~min}$. Fresh canine BMSCs at the second passage were used as a control $(n=6)$. Cell viability was assessed by trypan-blue 
exclusion in the DF and DF-free groups immediately after thawing and in the control group at the passage. In the DF group, cryopreserved cells were washed twice with $\alpha$-MEM containing $10 \%$ FBS before culture. Cells were seeded in 6-well plates at a density of $1 \times 10^{4}$ cells/well and cultured in $\alpha$-MEM containing $10 \%$ FBS; cell morphology was evaluated in cultures using an inverted microscope. Adherent cells were collected at days 2, 4, 6, 8, 10 and 12 of culture by dissociation with $0.25 \%$ trypsin-EDTA and counted to assess proliferative capacity.

Neuronal differentiation of canine BMSCs was induced by use of the method described by Woodbury et al. [21]. After 3 days of culture, the medium was changed to low-glucose Dulbecco's modified Eagle's medium (DMEM; Invitrogen) supplemented with $20 \%$ FBS and $1 \mathrm{mM} \beta$-mercaptoethanol (BME; Sigma-Aldrich). After $24 \mathrm{hr}$ of culturing in this medium, differentiation into neurons was induced using low-glucose DMEM with 2\% DMSO and $200 \mu \mathrm{M}$ butylated hydroxyanisole (BHA; Sigma-Aldrich), and morphological changes were evaluated by microscopy. After $6 \mathrm{hr}$ of neuronal induction, cells were fixed in $10 \%$ buffered formalin solution and reacted with the anti-human neurofilament light chain (NF-L) protein monoclonal antibody (Clone No. 2F11, Thermo Fisher Scientific Inc., Rockford, IL, U.S.A.) and anti-bovine microtubule-associated protein 2 (MAP-2) monoclonal antibody (Clone No. AP20, Millipore, Billerca, MA, U.S.A.) used as neuronal makers.

Total RNA was extracted from canine BMSCs using TRIzol (Invitrogen) before and $6 \mathrm{hr}$ after neuronal induction. The first-strand cDNA was synthesized using the PrimeScript RT Master Mix (Takara, Otsu, Japan). Quantitative PCR was performed with primers specific for genes expressed by neural stem/progenitor cells and neurons (Table 1). The thermal cycler was programmed to incubate initial denaturation for $30 \mathrm{sec}$ at $95^{\circ} \mathrm{C}$ and then to proceed with 40 cycles of primer annealing for $5 \mathrm{sec}$ at $95^{\circ} \mathrm{C}$ and primer extension for $30 \mathrm{sec}$ at $60^{\circ} \mathrm{C}$. The results were analyzed by the second derivative method and $\Delta \Delta \mathrm{Ct}$ method using TP900 DiceRealTime v4.02B (Takara). Glucuronidase $\beta$ (GUSB) was used as an endogenous control, and the amplification of the control group was used for calibration.

Statistical analysis of the data was performed using the StatMate IV software package (ATMS, Tokyo, Japan). The data were analyzed by two-way analysis of variance (ANOVA) among the groups, and Tukey's test was used as the post-hoc test. Paired student $t$-test was performed to compare the data before and after neuronal induction. The data are presented as means \pm standard errors; $P$ values less than 0.05 are considered significant.

The morphology of canine BMSCs in the DF and DF-free groups immediately after thawing was similar to that in the control group. No significant differences in cell numbers were observed between before and after cryopreservation in the DF and DF-free groups. The cell viability in the DF-free group $(99.0 \pm 0.9 \%)$ was higher than in the DF group $(89.7$ $\pm 3.0 \%)$ and almost the same as in the control group (100\%). Proliferation of fibroblast-like cells was observed in both DF and DF-free groups. But, while the proliferative capacity in the DF-free group was similar to that in the control group, it was lower in the DF group despite morphological similarity of the cells in all the groups (Fig. 1).

After neuronal induction, cryopreserved canine BMSCs in both the DF and DF-free groups developed neuron-like morphology (Fig. 2A and 2B), and nearly all neuron-like cells stained positive for NF-L and MAP-2 (Fig. 2D, 2E, 2G and $2 \mathrm{H}$ ), similar to the control group (Fig. 2C, 2F and 2I). The positive ratio of NF-L or MAP-2 was approximately $90 \%$ in each group, and no significant differences in the ratio were observed among the groups. Cryopreserved canine BMSCs expressed mRNA of sex determining region Y-box 2 $(S O X-2)$, nestin $(N E S)$ and POU domain transcription factor 1 (POU5F1) genes for neural stem/progenitor cell makers after thawing, and no significant differences were observed between the DF and DF-free groups. Upon neuronal induction, the mRNA expression levels for $M A P-2$ were significantly increased in all groups (Fig. 2J). MAP-2 mRNA expression level after neuronal induction in the DF-free group was significantly higher than in the DF and control groups (Fig. 2J). The mRNA expression levels for neurofilament$\mathrm{L}$ polypeptide $(N E F L)$ were also increased after neuronal induction in all groups (Fig. 2K).

In the present study, cryopreserved canine BMSCs demonstrated high cell viability and proliferative capacity after thawing and expressed mRNA for stem cell makers. These results suggest that cryopreservation of canine BMSCs using this method might not affect these capacities and expressions. To the best of our knowledge, neuronal differentiation potential of cryopreserved canine BMSCs has not been previously investigated. In the present study, we showed that upon neuronal induction, the cryopreserved canine BMSCs acquired neuron-like morphology and stained positive for neuronal marker MAP-2 and NF-L; in addition, the mRNA expression levels for neuronal markers $M A P-2$ and $N E F L$ were increased. These results suggest that cryopreserved canine BMSCs with this method might have the capacity to differentiate into a neuronal lineage.

In the present study, the positive ratios of neuron markers were approximately $90 \%$ after neuronal induction in each group, and these results were similar to other and our previous in vitro studies using the same neural induction protocol $[2,14,21]$. However, the ratio of the cells that changed to the neuron-like morphology was much lower than the positive ratios of neuron markers. It has been reported that BMSCs are not composed of a single cell type but of crude heterogenous cell populations and in vivo differentiation potential of BMSCs into neuron is generally low [8]. From these reasons, it is questionable whether approximately $90 \%$ of cryopreseved canine BMSCs differentiate into functional, mature neurons in vivo. In addition, Lu et al. suggested that the morphological changes and immunocytochemistry were simply artifacts after using chemical induction with $\mathrm{BME}$ and BHA to induce the development of rat BMSCs into neurons [14]. Therefore, additional investigations using electrophysiological techniques should be performed to prove that cryopreserved canine BMSCs are able to differentiate into functional, mature neurons. Further investigations, 
Table 1. Primers canine specific for neural stem/progenitor cell and neuron markers

\begin{tabular}{llll}
\hline Gene symbol & \multicolumn{1}{c}{ Protein } & \multicolumn{1}{c}{ Sequence } & Marker \\
\hline GUSB & Glucuronidase $\beta$ & F: ACATCGACGACATCACCGTCA & Housekeeping gene \\
& & R: GGAAGTGTTCACTGCCCTGGA & \\
\hline SOX-2 & Sex determining region Y-box 2 & F: ATGCACCGCTACGACGTGA & Neural stem/progenitor cell \\
& & R: TGCTGCGAGTAGGACATGCTG & \\
NES & Nestin & F: GGACGGGCTTGGTGTCAATAG & \\
& & R: AGACTGCTGCAGCCCATTCA & \\
POU5F1 & Oct-3/4 & F: TGCCAAGCTCCTGAAGCAGA & \\
& & R: AAACTGAGCTGCAGAGCCTCAA & \\
\hline MAP-2 & Microtubule-associated protein 2 & F: AAGCATCAACCTGCTCGAATCC & Neuron \\
& & R: GCTTAGCGAGTGCAGCAGTGAC & \\
NEFL & Neurofilament light chain & F: TGAATATCATGGGCAGAAGTGGAA & \\
& & R: GGTCAGGATTGCAGGCAACA & \\
\hline
\end{tabular}

A

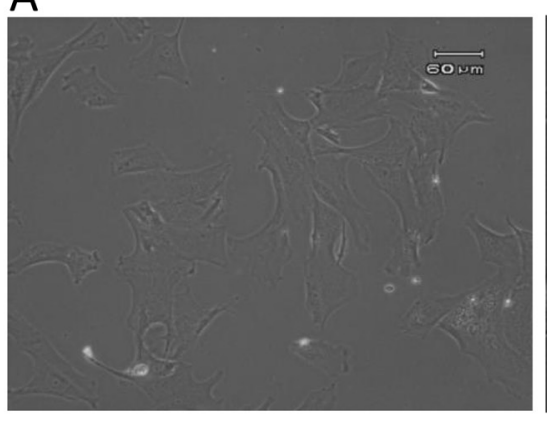

B

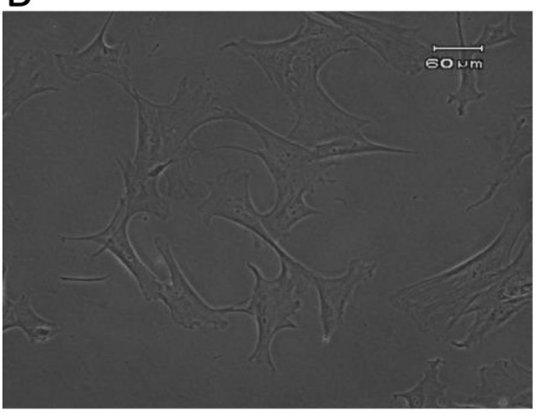

C

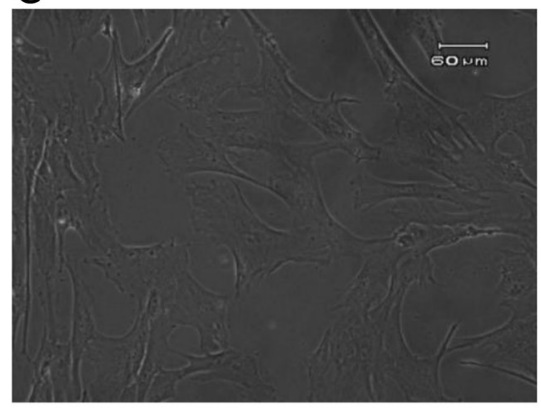

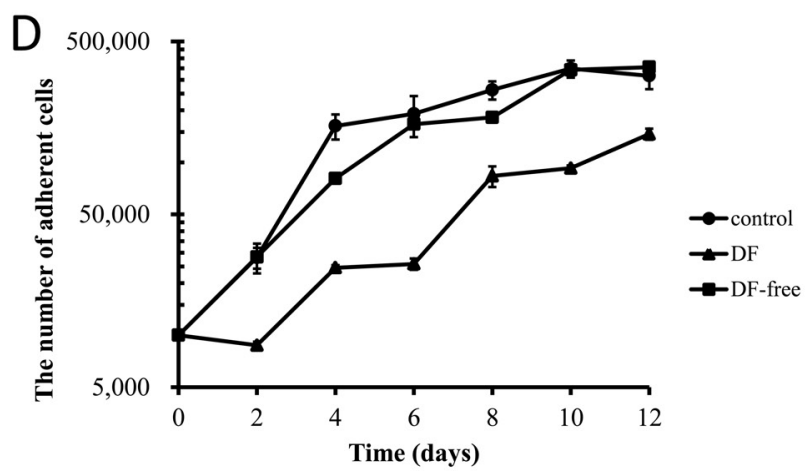

Fig. 1. Morphology of canine bone marrow stromal cells (BMSCs) in the DF (A), DF-free (B) and control (C) groups. (D) The proliferative capacity of canine BMSCs in the DF (closed triangles), DF-free (closed boxes) and control (closed circles) groups.

including in vivo experiments, will also be needed, before cryopreserved canine BMSCs are clinically applied to dogs with SCI.

The selection of an optimal cryoprotectant and freezing method is the key to the successful cryopreservation of canine BMSCs. There have been few reports on the cryopreservation of canine BMSCs $[10,11]$. In those studies, a cryoprotectant solution containing $10 \%$ DMSO and $10 \%$ FBS or newborn calf serum was used $[10,11]$. However, DMSO showed both cytotoxicity and neurological toxicity $[3,5,6,13,20]$, and FBS could cause bovine-derived infec- tious diseases and heteroantigen-induced immune reactivity. It is thus important to develop a cryoprotectant solution without DMSO and/or FBS for the cryopreservation of canine BMSCs. Our results demonstrate that the cell viability and proliferative capacity of canine BMSCs in the DF-free group were superior to those in the DF group. Furthermore, upon neuronal induction, $M A P-2$ mRNA expression in the DF-free group was significantly higher than in the DF group. The DMSO/FBS-free cryoprotectant solution used in this study may be beneficial for cryopreservation of canine BM$\mathrm{SCs}$ for subsequent use in spinal cord regenerative therapy. 

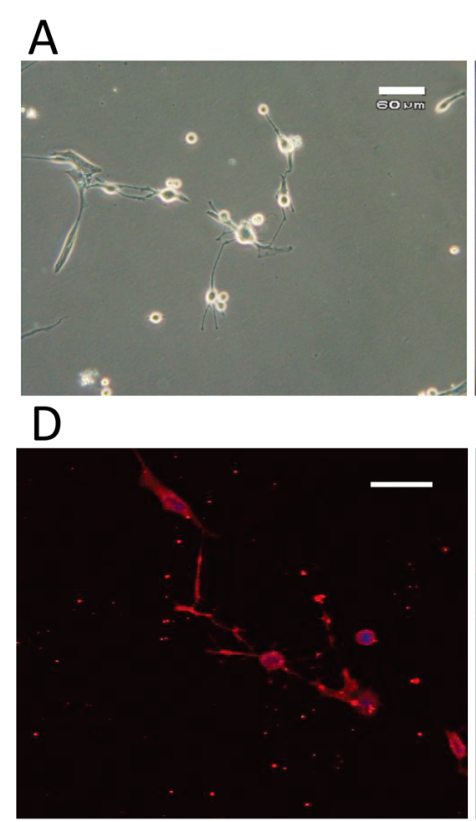

G

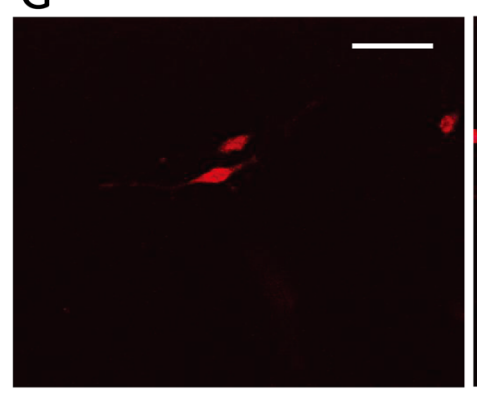

J

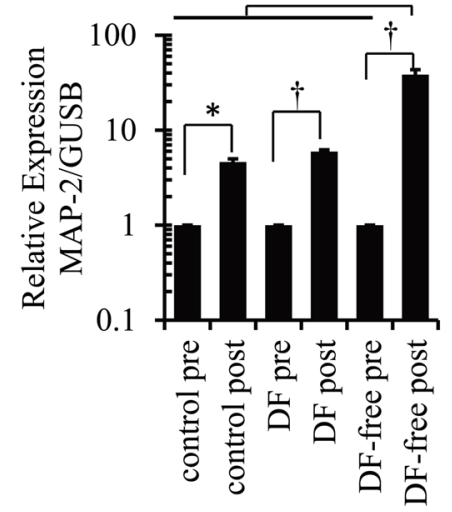

B

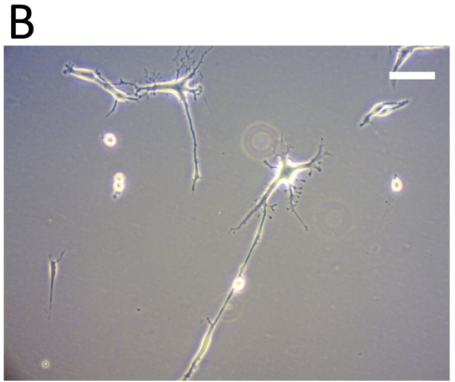

E

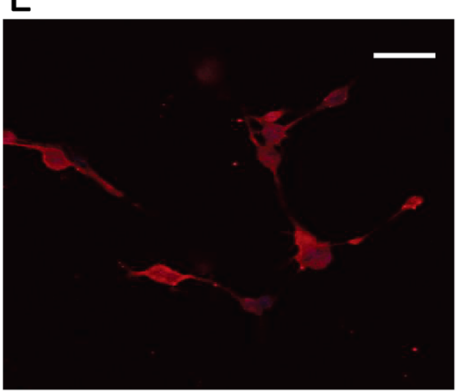

$\mathrm{H}$

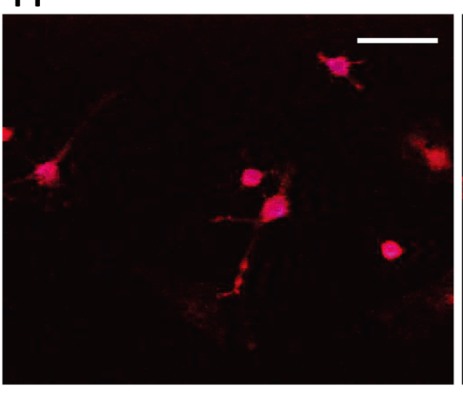

K

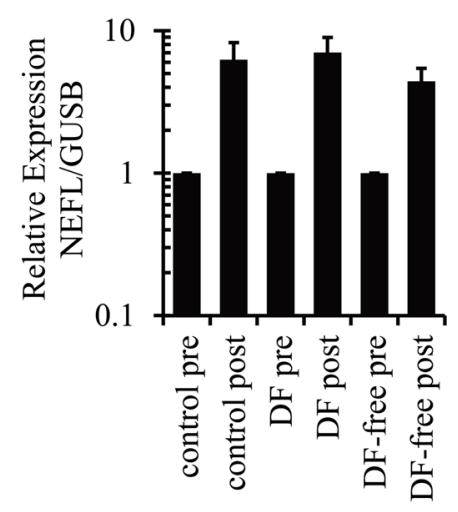

C

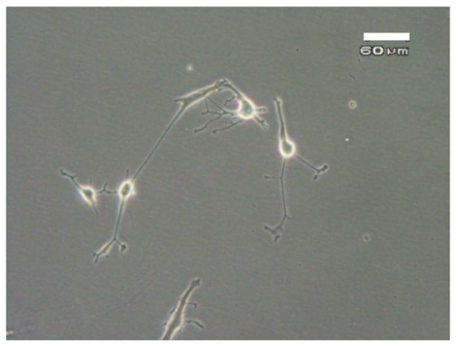

$\mathrm{F}$

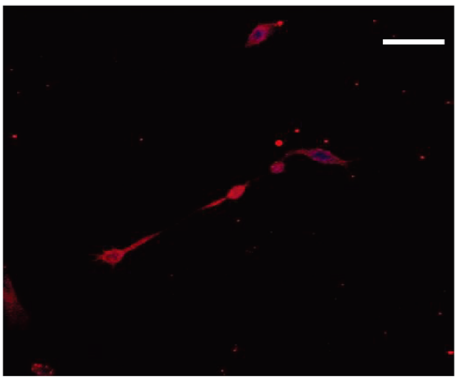

I

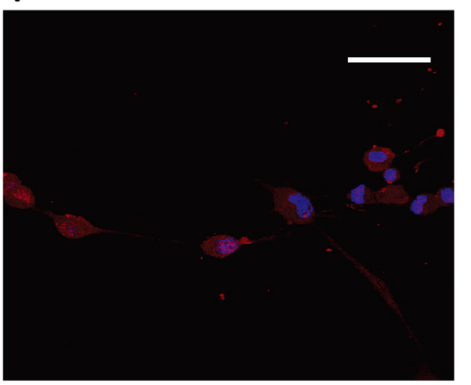

Fig. 2. Morphology of canine BMSCs in the DF (A), DF-free (B) and control (C) groups at $6 \mathrm{hr}$ after neuronal induction. Canine BMSCs developed neuron-like morphology in all groups. Scale bars $=60 \mu \mathrm{m}(\mathrm{A}-\mathrm{C})$. Immunocytochemical staining of neuron-like cells in the DF (D, G), DF-free (E, H) and control (F, I) groups; these neuron-like cells were positive for MAP-2 (D-F) and neurofilament-L (G-I). Scale bars $=30 \mu \mathrm{m}$ (D-I). The mRNA expression levels of MAP$2(\mathrm{~J})$ and $N E F L(\mathrm{~K})$ before (pre) and $6 \mathrm{hr}$ after (post) neuronal induction. (J) Significant increases were detected in all groups (*: $P<0.05, \uparrow: P<0.01, \dagger \dagger: P<0.001)$.

Another problem in the cryopreservation of stem cells is that costly equipment and complicated procedures are usually required. Simple methods must be established in order to popularize the cryopreservation of canine BMSCs in a clinical setting. BMSCs are commonly frozen using a programmable freezer or multi-step freezing procedure $[7,10$, 
11]. In the present study, instead of a programmable freezer, we used a biofreezing vessel, which ensured slow freezing. The cooling rate of the biofreezing vessel used in the present study is approximately $-1.0^{\circ} \mathrm{C} / \mathrm{min}$, and the biofreezing vessel is able to provide an environment similar to a programmable freezer $[9,19]$. Several studies have documented that conventional controlled-rate freezing and storage in liquid nitrogen are not absolutely necessary for a successful cryopreservation of human progenitor cells and could be replaced by storage at $-80^{\circ} \mathrm{C}$ in a mechanical freezer $[1,15]$. The biofreezing vessel used in this study is designed to be able to store the cells at $-80^{\circ} \mathrm{C}$. In our case, the biofreezing vessel was placed in a deep freezer, a very simple procedure that does not require specialized equipment, although further investigations including the effects of long-term cryopreservation are necessary.

In conclusion, cryopreservation showed no significant adverse effects on the cell viability, proliferative capacity and differentiation potential into a neuronal lineage of canine BMSCs. Our cryopreservation method using DF-free cryoprotectant solution and a biofreezing vessel may be very useful for cryopreservation of canine BMSCs and subsequent spinal cord regenerative therapy in dogs.

ACKNOWLEDGMENT. This study was supported in part by the Grant-in-Aid for Young Scientists (B; 18780240) from the Ministry of Education, Science, Sports and Culture of Japan.

\section{REFERENCES}

1. Calvet, L., Cabrespine, A., Boiret-Dupré, N., Merlin, E., Paillard, C., Berger, M., Bay, J. O., Tournilhac, O. and Halle, P. 2013. Hematologic, immunologic reconstitution, and outcome of 342 autologous peripheral blood stem cell transplantations after cryopreservation in $\mathrm{a}-80^{\circ} \mathrm{C}$ mechanical freezer and preserved less than 6 months. Transfusion 53: 570-578. [Medline] [CrossRef]

2. Edamura, K., Kuriyama, K., Kato, K., Nakano, R., Teshima, K., Asano, K., Sato, T. and Tanaka, S. 2012. Proliferation capacity, neuronal differentiation potency and microstructures after the differentiation of canine bone marrow stromal cells into neurons. J. Vet. Med. Sci. 74: 923-927. [Medline] [CrossRef]

3. Haack-Sørensen, M. and Kastrup, J. 2011. Cryopreservation and revival of mesenchymal stromal cells. pp.161-174. In: Mesenchymal stem cell assays and applications. (Vemuri, M., Chase, L.G. and Rao, S. eds.), Humana press, New York.

4. Heng, B. C. 2009. Effect of Rho-associated kinase (ROCK) inhibitor Y-27632 on the post-thaw viability of cryopreserved human bone marrow-derived mesenchymal stem cells. Tissue Cell 41: 376-380. [Medline] [CrossRef]

5. Hunt, C. J. 2011. Cryopreservation of human stem cells for clinical application: A review. Transfus. Med. Hemother. 38: 107-123. [Medline] [CrossRef]

6. Júnior, A. M., Arrais, C. A., Saboya, R., Velasques, R. D., Junqueira, P. L. and Dulley, F. L. 2008. Neurotoxicity associated with dimethylsulfoxide-preserved hematopoietic progenitor cell infusion. Bone Marrow Transplant. 41: 95-96. [Medline] [CrossRef]

7. Kotobuki, N., Hirose, M., Machida, H., Katou, Y., Muraki, K., Takakura, Y. and Ohgushi, H. 2005. Viability and osteogenic potential of cryopreserved human bone marrow-derived mesenchymal cells. Tissue Eng. 11: 663-673. [Medline] [CrossRef]

8. Kuroda, Y., Wakao, S., Kitada, M., Murakami, T., Nojima, M. and Dezawa, M. 2013. Isolation, culture and evaluation of multilineage-differentiating stress-enduring (Muse) cells. Nat. Protoc. 8: 1391-1415. [Medline] [CrossRef]

9. Lee, S., Iwasaki, Y., Shikina, S. and Yoshizaki, G. 2013. Generation of functional eggs and sperm from cryopreserved whole testes. Proc. Natl. Acad. Sci. U.S.A. 110: 1640-1645. [Medline] [CrossRef]

10. Li, H., Yan, F., Lei, L., Li, Y. and Xiao, Y. 2009. Application of autologous cryopreserved bone marrow mesenchymal stem cells for periodontal regeneration in dogs. Cells Tissues Organs 190: 94-101. [Medline] [CrossRef]

11. Li, H. X., Yan, F. H., Lei, L. and Liu, J. G. 2008. Effect of cryopreservation of bone marrow stromal cells on periodontal tissue regeneration. Zhonghua Kou Qiang Yi Xue Za Zhi 43: 246-247. [Medline]

12. Liu, G., Zhou, H., Li, Y., Li, G., Cui, L., Liu, W. and Cao, Y. L. 2008. Evaluation of the viability and osteogenic differentiation of cryopreserved human adipose-derived stem cells. Cryobiology 57: 18-24. [Medline] [CrossRef]

13. Liu, Y., Xu, X., Ma, X., Martin-Rendon, E., Watt, S. and Cui, Z. 2010. Cryopreservation of human bone marrow-derived mesenchymal stem cells with reduced dimethylsulfoxide and welldefined freezing solutions. Biotechnol. Prog. 26: 1635-1643. [Medline] [CrossRef]

14. Lu, P., Blesch, A. and Tsuzynski, M. H. 2004. Induction of bone marrow stromal cells to neurons: differentiation, transdifferentiation, or artifact? J. Neurosci. Res. 77: 174-191. [Medline] [CrossRef]

15. McCullough, J., Haley, R., Clay, M., Hubel, A., Lindgren, B. and Moroff, G. 2010. Long-term storage of peripheral blood stem cells frozen and stored with a conventional liquid nitrogen technique compared with cells frozen and stored in a mechanical freezer. Transfusion 50: 808-819. [Medline] [CrossRef]

16. Nie, Y., Bergendahl, V., Hei, D. J., Jones, J. M. and Palecek, S. P. 2009. Scalable culture and cryopreservation of human embryonic stem cells on microcarriers. Biotechnol. Prog. 25: 20-31. [Medline] [CrossRef]

17. Nishida, H., Nakayama, M., Tanaka, H., Kitamura, M., Hatoya, S., Sugiura, K., Suzuki, Y., Ide, C. and Inaba, T. 2011. Evaluation of transplantation of autologous bone marrow stromal cells into the cerebrospinal fluid for treatment of chronic spinal cord injury in dogs. Am. J. Vet. Res. 72: 1118-1123. [Medline] [CrossRef]

18. Nishida, H., Nakayama, M., Tanaka, H., Kitamura, M., Hatoya, S., Sugiura, K., Harada, Y., Suzuki, Y., Ide, C. and Inaba, T. 2012. Safety of autologous bone marrow stromal cell transplantation in dogs with acute spinal cord injury. Vet. Surg. 41: 437-442. [Medline] [CrossRef]

19. Ogonuki, N., Mochida, K., Miki, H., Inoue, K., Fray, M., Iwaki, T., Moriwaki, K., Obata, Y., Morozumi, K., Yanagimachi, R. and Ogura, A. 2006. Spermatozoa and spermatids retrieved from frozen reproductive organs or frozen whole bodies of male mice can produce normal offspring. Proc. Natl. Acad. Sci. U.S.A. 103: 13098-13103. [Medline] [CrossRef]

20. Thirumala, S., Goebel, W. S. and Woods, E. J. 2009. Clinical grade adult stem cell banking. Organogenesis 5: 143-154. [Medline] [CrossRef]

21. Woodbury, D., Schwarz, E. J., Prockop, D. J. and Black, I. B. 2000. Adult rat and human bone marrow stromal cells differentiate into neurons. J. Neurosci. Res. 61: 364-370. [Medline] [CrossRef] 